\title{
Desempeño de reactores empacados con marlo de maíz (Zea mays) en la remoción de hierro contenido en drenajes ácidos de mina
}

\section{Performance of packed - bed corncob (Zea mays) in the removal of iron contained in acid mine drainage}

\author{
Angie Lorena Hernández Botia ${ }^{\mathrm{a}^{*}}$ \\ Olga Yaneth Vásquez Ochoa ${ }^{b}$ \\ Fabio Augusto Roldan García ${ }^{\mathrm{c}}$ \\ Jairo Antonio Cubillos Lobo ${ }^{\mathrm{d}}$ \\ Gerardo Andrés Caicedo Pineda ${ }^{\mathrm{e}}$
}

Fecha de Recepción: 12.12.2019

Fecha de Aceptación: 15.04.2020

Doi: https://doi.org/10.19053/01217488.v11.n2.2020.11279

\section{Resumen}

Se evaluó la capacidad del marlo de maíz (Zea mays) para remover hierro contenido en drenajes ácidos de mina (DAM) de carbón, en reactores de lecho empacado, a escala laboratorio. Los ensayos tuvieron una fase de filtrado de efluente, a través del lecho, a un caudal de $5 \mathrm{~mL} \times \mathrm{min}^{-1}$ y un lavado de columna, con agua destilada, a fin de remover lo débilmente retenido por la biomasa. Las variables evaluadas fueron: (i) modificación del marlo con hidróxido de sodio $(\mathrm{NaOH})$ o ácido cítrico $\left(\mathrm{C}_{6} \mathrm{H}_{8} \mathrm{O}_{7}\right)$ y (ii) concentración de hierro en el DAM de 500 ppm, 1,000 ppm y 2,000 ppm. Simultáneamente, se hicieron ensayos, bajo las mismas condiciones, pero, con lavados de agua destilada al marlo, cada $400 \mathrm{~mL}$ de DAM filtrado, con el fin de evitar la saturación del sistema. El efluente tratado se monitoreó cada 80 min, mediante mediciones de $\mathrm{pH}$ y hierro (espectroscopia de absorción atómica). Las biomasas se caracterizaron fisicoquímicamente, al iniciar y al finalizar el proceso, mediante espectroscopia infrarroja con transformada de Fourier (FTIR). En general, el pH del DAM jugó un papel muy importante en la capacidad de remoción del metal, en especial, cuando se utilizó concentraciones de Fe de 500 ppm, puesto que los protones de esta solución tienen una mayor competencia por los sitios activos de adsorción del lecho empacado, reduciendo así el área de superficie efectiva disponible. Lo anterior no ocurrió cuando se utilizó marlo modificado con $\mathrm{NaOH}$ y concentraciones de hierro superiores a $1,000 \mathrm{ppm}$, debido al incremento de colisiones entre el hierro y el lecho empacado de marlo de maíz, que mitigaría el efecto del pH. Los ensayos con lavados esporádicos durante el filtrado del DAM permitieron confirmar que la prolongación del tiempo de reúso de la biomasa, sólo es posible si se reduce dicho efecto. Esta investigación de remoción de hierro contenido en DAM con marlo de maíz, podría ser una alternativa para aportar parámetros de tratamiento de remediación de efluentes de una manera sencilla y económica que tal vez funcione en posteriores investigaciones que incluyan pretratamientos en

a Ingeniera Metalúrgica. Universidad Pedagógica y Tecnológica de Colombia, Avenida Central del Norte 39-115, angie. hernandez@uptc.edu.co (autor de correspondencia).

b PhD. Ciencias Biológicas. Universidad Central. Escuela de Química, Cl. 21 \#4-40, (Bogotá-Colombia), ovasquezo@ ucentral.edu.co.

PhD. Ingeniería Civil. Universidad Javeriana Cra.7\#No.40-62, (Bogotá-Colombia), fabio.roldan@javeriana.edu.co.

d PhD. Ciencias Naturales. Universidad Pedagógica y Tecnológica de Colombia, Avenida Central del Norte 39-115, Escuela de Ciencias Química (Tunja-Colombia), jairo.cubillos@uptc.edu.co.

e PhD. Ingeniería - Ciencia y Tecnología de los Materiales, Universidad Pedagógica y Tecnológica de Colombia, Avenida Central del Norte 39-115, Escuela de Ciencias Química (Tunja-Colombia), gerardo.caicedo@uptc.edu.co. 
donde empleen sistemas de remediación pasivos a nivel piloto o planta, contribuyendo así finamente con la disminución del impacto negativo de lixiviados contaminantes.

Palabras clave: Acondicionamiento químico, adsorción, biomasa, mina de carbón, remediación,

\begin{abstract}
The ability of corncob (Zea mays) to remove iron contained in acidic mine drains (AMD) from coal in laboratory-scale packed bed reactors was evaluated. The tests had an effluent filtering phase, through the bed, at a flow rate of $5 \mathrm{~mL} \times \mathrm{min}^{-1}$ and a column wash, with distilled water, in order to remove weakly adsorbed material. The variables evaluated were: (i) modification of the corncob with sodium hydroxide $(\mathrm{NaOH})$ or citric acid $\left(\mathrm{C}_{6} \mathrm{H}_{8} \mathrm{O}_{7}\right)$ and (ii) iron concentration in the AMD of $500 \mathrm{ppm}, 1,000 \mathrm{ppm}$ and 2,000 ppm. At the same time, trials were performed at the same conditions, but, with washes of distilled water to the corncob, each $400 \mathrm{~mL}$ of filtered AMD, in order to avoid saturation of the system. The treated effluent was monitored every $80 \mathrm{~min}$ through $\mathrm{pH}$ and iron measurements (atomic absorption spectroscopy). The biomasses were physicochemically characterized at the beginning and at the end of the process using FTIR. Overall, the $\mathrm{pH}$ of AMD played an key role in the metal removal capacity, mainly when iron concentrations of $500 \mathrm{Fe} \mathrm{ppm}$ were used, since the protons of this solution have a greater competition for the active adsorption sites in the packed bed, thus reducing the available effective surface area. The previous did not occur when using modified corncob with $\mathrm{NaOH}$ and iron concentrations higher than 1,000 ppm, due to the increased collisions between iron and the packed bed of corncob, which would mitigate the effect of $\mathrm{pH}$. The tests with sporadic washes during the AMD filtering confirmed that the prolongation of the biomass reuse time is only possible if this effect is reduced. This work that pointed to the removal of iron contained in AMD with chemically modified corncob, could be an alternative to provide parameters of effluent remediation treatment in a simple and economical way that may be coupled to other passive remediation systems at greater scale, thus contributing to the reduction of the negative impact of contaminating leachates.
\end{abstract}

Keywords: Chemical conditioning, adsorption, biomass, coal mine, remediation.

\section{INTRODUCCIÓN}

El marlo es un material de desecho lignocelulósico, procedente de la planta de maíz [1,2]. Generalmente, se encuentra en abundancia y se desperdicia o se quema, causando contaminación ambiental sin ningún provecho [3]. Se compone principalmente de celulosa, hemicelulosa, lignina, ceniza y pectina $[4,5]$. Desde hace décadas, investigaciones acerca de tratamientos de efluentes contaminados con biomasas, comprueban que esta clase de materiales pueden tener valor agregado, ya que funcionan como biosorbentes de bajo costo, para la remoción de detergentes, colorantes, sales, aceite, grasa y metales pesados en solución [6-8]. Sus componentes estructurales proteínicos, polisacáridos o nucleótidos, que a su vez contienen grupos funcionales como carbonilos, carboxilos, hidroxilos y aminos y su estructura física irregular, porosa y con buena área superficial, le confieren la capacidad de atrapamiento de partículas en suspensión sobre su biomasa, mediante procesos de complejación, adsorción física e intercambio iónico [2-4,6].

La unión marlo - metal depende de la morfología, estructura composicional y propiedades químicas de la biomasa y del metal, así como también de las propiedades químicas, temperatura y fuerza iónica del medio [1]. Piboon et al., (2017) demostrado que el tratamientos de remediación de efluentes por métodos de modificación con alcalinizantes fuertes o ácidos orgánicos, pueden alterar la estructura heterogénea de las biomasas, adicionando grupos funcionales que disminuyen la cristalinidad e incrementan el área superficial y la porosidad, por tanto confiriéndole mejores propiedades de remoción de metales [9].

Peñaranda O. et al., (2008), en su estudio de modificación química de almidones, afirman que al modificar biomasas con $\mathrm{NaOH}$ se favorece la disposición de grupos hidroxilos dentro de la estructura del marlo y se aumenta el área superficial interna, generando una morfología idónea para el atrapamiento de metales [10]. En otra investigación, Feng et al., (2010) indican que la estructura lignocelulósica de la biomasa contiene ésteres metílicos que se transforman a ligandos de carboxilato, luego de una modificación con $\mathrm{NaOH}$, haciendo que se aumente la capacidad de unión biomasa-metal [11] . En la Ec. (1) se muestra la reacción de hidrólisis básica que sufre dichos ésteres metílicos: 


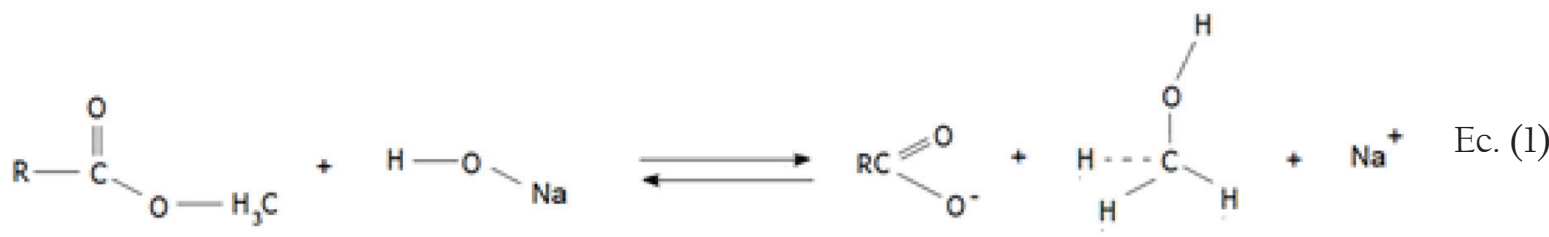

Por otra parte, Goyal et al., (2007) en su investigación acera de la caracterización de la biomasa de maíz modificada con ácido cítrico $\left(\mathrm{C}_{6} \mathrm{H}_{8} \mathrm{O}_{7}\right)$ afirma que se obtienen biomateriales eficaces para la remoción de metales, prolongando su ciclo reutilizable [12] [13]. Se le atribuye al anión carboxilato del grupo ácido su capacidad de unión de cationes en la biomasa, ya que este se convierte en un sitio activo para el atrapamiento de los metales
[14]. En la Ec. (2) se observa la modificación de la fibra de maíz con $\mathrm{C}_{6} \mathrm{H}_{8} \mathrm{O}_{7}$, mediante un proceso de carboxilación, en donde el ácido por calentamiento produce un anhídrido cítrico el cual, al estar en mezcla con la biomasa forma un éster, adiciona grupos carboxílicos que posteriormente interactuarán con los iones metálicos de la solución a tratar [15].<smiles>[R20]C(=O)CC(CC(=O)O)(O[C@H](O)C(O)O)C(=O)O</smiles>

Teniendo en cuenta lo expuesto, la biomasa del marlo de maíz se muestra como una alternativa atractiva en la remoción de metales en medios acuosos ya que es abundante, variable, de bajo costo y de fácil acceso [4]. Este tipo de tratamientos podría contribuir con alternativas de solución al gremio minero que mayoritariamente se encuentra interesado en incorporar métodos que minimicen los impactos ambientales generados por los drenajes ácidos de mina (DAM) de una manera económica, sustentable y ambientalmente favorable.

Los DAM de la minería de metales y carbón se consideran una de las principales fuentes de contaminación de los recursos hídricos y suelen ser peligrosos según sus constituyentes metálicos, representando así una amenaza para los ecosistemas y la salud de las personas [16]. Se caracterizan por tener bajos $\mathrm{pH}$ y una alta concentración de hierro. Ellos se originan de minas que contienen rocas sulfuradas que al entrar en contacto con el oxígeno y el agua del ambiente, reducen la solubilidad del hierro y liberan ácidos minerales permitiendo la disolución de metales [17].
En general los estudios que emplean marlo de maíz para la remoción de metales en solución se han evaluado a escala de laboratorio (10-50 $\mathrm{mL})$, utilizando sistemas "ideales" que permiten uniformidad de las reacciones y reducen la ocurrencia de reacciones secundarias que afectan el proceso [18]. Estos reactores tipo "batch" permiten el control de factores como composición, temperatura y presión durante el tratamiento. Sin embargo, aunque son sistemas comunes en las industrias tienden a ser costosos y no se consideran viables en el tratamiento de aguas, ya que se requiere tratar volúmenes considerables [19].

Este panorama suscita analizar la eficiencia de un lecho empacado con marlo nativo y modificado con $\mathrm{NaOH}$ y $\mathrm{C}_{6} \mathrm{H}_{8} \mathrm{O}_{7}$ para la remoción de hierro contenido en DAM preparados a nivel laboratorio con características muy similares a los drenajes provenientes de la mina de carbón Milpa-2 de la empresa INCARSA LTDA del municipio de Samacá - Boyacá. La evaluación de la eficiencia se hizo mediante el análisis de las interacciones fisicoquímicas marlo - hierro, mientras que la remoción de hierro del DAM se determinó por 
espectroscopia de absorción atómica (EAA) y los cambios estructurales en la biomasa utilizada por espectroscopia infrarroja con transformada de Fourier (FTIR). Finalmente, la estimación de remoción de hierro en el lecho se hizo acorde a los resultados que se obtuvieron en cada uno de los procesos llevados a cabo.

\section{MATERIALES Y MÉTODOS}

\section{Preparación y modificación del marlo de maíz:}

Se recolectó $12 \mathrm{Kg}$ de marlo de maíz en una plaza de mercado ubicada en el sur de la ciudad de Tunja capital de Boyacá (Colombia). Se lavó con abundante agua, para eliminar impurezas y se secó a temperatura ambiente por 3 días. Posteriormente, se trituró a un tamaño de partícula $-2+30$ (según la serie de tamices Tyler) y, mediante la técnica de cuarteo, se seleccionaron muestras de aproximadamente $2 \mathrm{Kg}$ para los diferentes procesos experimentales.

Las modificaciones del marlo de maíz se llevaron a cabo de acuerdo con el procedimiento propuesto por Altún \& Pehlivan (2012) [20]. Inicialmente, se trató la biomasa con una solución de $\mathrm{NaOH} 1 \mathrm{M}$ en una proporción $0.4 \mathrm{~g}$ de marlo a $1.0 \mathrm{~mL}$ de solución, llevando la mezcla a una temperatura de $60^{\circ} \mathrm{C}$ por $24 \mathrm{~h}$. Finalmente, se lavó el producto con agua desionizada y se secó por $24 \mathrm{~h}$ a $55^{\circ} \mathrm{C}$. Siguiendo el mimo procedimiento anterior, se modificó el marlo con $\mathrm{C}_{6} \mathrm{H}_{8} \mathrm{O}_{7}$, cambiando la solución de $\mathrm{NaOH} 1 \mathrm{M}$ por $\mathrm{C}_{6} \mathrm{H}_{8} \mathrm{O}_{7} 1 \mathrm{M}[2,18]$.

El marlo de maíz con y sin modificación se caracterizó mediante mediciones de $\mathrm{pH}$ en solución acuosa, humedad, densidad aparente, densidad real y porosidad siguiendo los procedimientos descritos por Pintado (2018) [21]. Además, se calculó el porcentaje de incorporación del modificante $(\%$ $I M)$ en la biomasa, para lo cual se tomó $4 \mathrm{~g}$ de marlo y se suspendió en $50 \mathrm{~mL}$ de agua, llevándose a agitación a $100 \mathrm{rpm}$ durante $30 \mathrm{~min}$. El sólido obtenido se recuperó mediante filtración por gravedad, se lavó con agua y se secó a $105^{\circ} \mathrm{C}$. El \% $I M$ se calcula con la Ec. (3), donde MA representa la cantidad en $\mathrm{g}$ de modificante agregado y MR la cantidad en g del modificante recuperado [14].

$$
\% \text { IM } \frac{\mathrm{MA}-\mathrm{MR}}{\mathrm{MA}} * 100 \% \quad \text { Ec. (3) }
$$

Por otra parte, la modificación de marlo de maíz se analizó por microscopía electrónica de barrido (SEM), con un equipo Leica - Zeiss LEO 440, tomando micrografías con un transformador de tensión extra alta (EHT) de $20 \mathrm{kV}$, un detector tipo $\mathrm{I}$ (SE1) a una distancia de trabajo de $9 \mathrm{~mm}$, con una escala de $20 \mu \mathrm{m}$. También, se determinó cambios composicionales, mediante Espectroscopia Infrarroja con Transformada de Fourier (FTIR), con un equipo Thermo Scientific Nicolet 5300, mediante la técnica de Reflectancia Total Atenuada (ATR), generando espectrogramas con un rango espectral entre 4,000 a $400 \mathrm{~cm}^{-1}$ a una resolución de $4 \mathrm{~cm}^{-1}$ con 32 barridos.

\section{Preparación del drenaje ácido de mina (DAM)}

El DAM se preparó, teniendo en cuenta el rango histórico de concentración de hierro y $\mathrm{pH}$ de efluentes provenientes una mina de carbón del municipio de Samacá (Boyacá) y lo establecido por Zagury et al. (2006). Para esto, se dejó $2 \mathrm{~L}$ de agua en reposo por $24 \mathrm{~h}$, luego se le añadió 1.074 $\mathrm{g} \mathrm{de} \mathrm{CaCO}_{3}$ y se agitó durante $12 \mathrm{~h}$. Seguidamente, se adicionó el $\mathrm{FeSO}_{4} \cdot 7 \mathrm{H}_{2} \mathrm{O}$ (II) (comercial) bajo agitación continua por $24 \mathrm{~h}$ hasta obtener la concentración deseada de hierro (2,000 ppm, 1,000 ppm y 500 ppm). Finalmente, se dejó en reposo y se ajustó el pH del DAM a 2.7, empleando una solución acuosa de $\mathrm{NaOH} 1 \mathrm{M}$ [22].

\section{Ensayos en biorreactores de lecho empacado}

Se utilizó un sistema de lecho empacado, el cual constaba de 3 columnas en acrílico de $20 \mathrm{~cm}$ de largo por $5 \mathrm{~cm}$ de diámetro, con su respectiva conexión de mangueras, empaques y recipientes de recolecta. La biomasa estuvo contenida entre 2 camas de algodón, de aproximadamente $2 \mathrm{~cm}$ de espesor, las cuales sirvieron tanto para evitar la obstrucción del sistema, como para garantizar una distribución uniforme del DAM a través del lecho empacado. Cada lecho empacado fue de $40 \mathrm{~g}$ de biomasa, compuesto por marlo de maíz modificado (con $\mathrm{NaOH}$ y $\mathrm{C}_{6} \mathrm{H}_{8} \mathrm{O}_{7}$ ) o sin modificar, por el cual se filtró DAM sintético, a tres concentraciones de hierro: 500 ppm, 1,000 ppm y 2,000 ppm.

Todos los ensayos se hicieron por triplicado y tuvieron 3 etapas de proceso: (i) empaque y adecuación del marlo, a fin de eliminar posibles 
Angie Lorena Hernández Botia - Olga Yaneth Vásquez Ochoa - Fabio Augusto Roldan García Jairo Antonio Cubillos Lobo - Gerardo Andrés Caicedo Pineda

agentes solubles que hayan resultado en su modificación, (ii) fase de filtrado, donde se pasaron 2,000 $\mathrm{mL}$ de DAM a través del lecho, a un caudal de $5 \mathrm{~mL} \times \mathrm{min}^{-1}$ y (iii) lavado de columna con agua destilada, luego de culminar la primera etapa, a fin de remover los metales que no fueron retenidos en la biomasa.

Adicionalmente, se ejecutaron ensayos con similitud a los anteriores, pero, con lavados del marlo de maíz con agua destilada, cada vez que pasaban $400 \mathrm{~mL}$ de efluente por la columna.

Todos los experimentos fueron monitoreados cada $400 \mathrm{~mL}$ mediante mediciones de $\mathrm{pH}$, utilizando un $\mathrm{pH}$-metro marca 850 SI ANALYTICS y análisis de concentración de hierro en solución por espectroscopia de absorción atómica (EAA), utilizando un equipo marca Shimadzu modelo AA 7000, mediante el método 7000B-USEPA (con límite de detección de 0.03-4.0 ppm) [23]. Al finalizar los procesos de filtración, el marlo de maíz se analizó por espectroscopia infrarroja con transformada de Fourier (FTIR), mediante la técnica de Reflectancia Total Atenuada (ATR).

\section{RESULTADOS}

\section{Caracterización del marlo de maíz con y sin modificación química}

En la Tabla (1) se muestra el consolidado de resultados obtenidos en la caracterización del marlo de maíz con y sin modificación química. Se evidencia que la biomasa aumenta su $\mathrm{pH}$ al ser modificada con $\mathrm{NaOH}$, mientras que disminuye cuando es modificada con $\mathrm{C}_{6} \mathrm{H}_{8} \mathrm{O}_{7}$. Así mismo, se observa que la modificación química del marlo de maíz incrementó la humedad, densidad y porosidad. Adicionalmente, el porcentaje de incorporación del $\mathrm{NaOH}$ y el $\mathrm{C}_{6} \mathrm{H}_{8} \mathrm{O}_{7}$ fue de aproximadamente de un $90 \%$ para ambos casos.

Tabla 1. Propiedades fisicoquímicas del marlo de maíz con y sin modificación química

\begin{tabular}{|c|c|c|c|c|}
\hline Característica & Nativo & Mod. $\mathrm{NaOH}$ & Mod. $\mathrm{C}_{6} \mathrm{H}_{8} \mathrm{O}_{7}$ & $p$ Valor \\
\hline pH & $5.45 \pm 0.06$ & $10.54 \pm 0.09$ & $2.53 \pm 0.15$ & $<0.0001$ \\
\hline Humedad (\%) & $15.13 \pm 0.98$ & $18.62 \pm 0.85$ & $16.47 \pm 0.71$ & 0.007 \\
\hline Densidad aparente $\left(g \times \mathrm{mL}^{-1}\right)$ & $0.32 \pm 0.04$ & $0.68 \pm 0.06$ & $0.55 \pm 0.05$ & 0.0004 \\
\hline Densidad real $g \times m L^{-1}$ ) & $0.78 \pm 0.05$ & $1.91 \pm 0.07$ & $1.51 \pm 0.03$ & $<0.0001$ \\
\hline Porosidad (\%) & $59 \pm 5.00$ & $64 \pm 5.00$ & $63 \pm 5.00$ & 0.4116 \\
\hline Incorporación del modificante (\%) & - & $91.31 \pm 1.77$ & $89.95 \pm 1.56$ & 0.4989 \\
\hline
\end{tabular}

Por otra parte, la Figura (1) representa las micrografias del marlo de maíz con y sin modificación química. En general, los resultados coinciden con el de otras investigaciones, donde caracterizan biomasas de productos agricolas en su estado natural y modificadas con $\mathrm{NaOH}$ y $\mathrm{C}_{6} \mathrm{H}_{8} \mathrm{O}_{7}$. Se reporta que su morfología es totalmente adecuada para la retención, atrapamiento y adsorción de iones metalico. En el caso del marlo nativo (Figura 1a), se evidenció una morfología heterogénea, rugosa $\mathrm{y}$ de poros abiertos con una estructura en forma de panal de abejas, con celdillas hexagonales confor- madas por una diversidad de láminas plegadas que se unen unas a otras, formando pequeños prismas de manera repetitiva.

La micrografia de la Figura (1b) corresponde al marlo de maíz modificado con $\mathrm{NaOH}$. Se observó una estructura cubierta por el modificante de manera heterogénea e imperfecta. El escaneo de la Figura (1c) pertenece al marlo de maíz modificado con $\mathrm{C}_{6} \mathrm{H}_{8} \mathrm{O}_{7}$, donde se evidenciaron estructuras fibrosas, cilíndricas heterogéneas con fomas y tamaños regulares y fijos. 

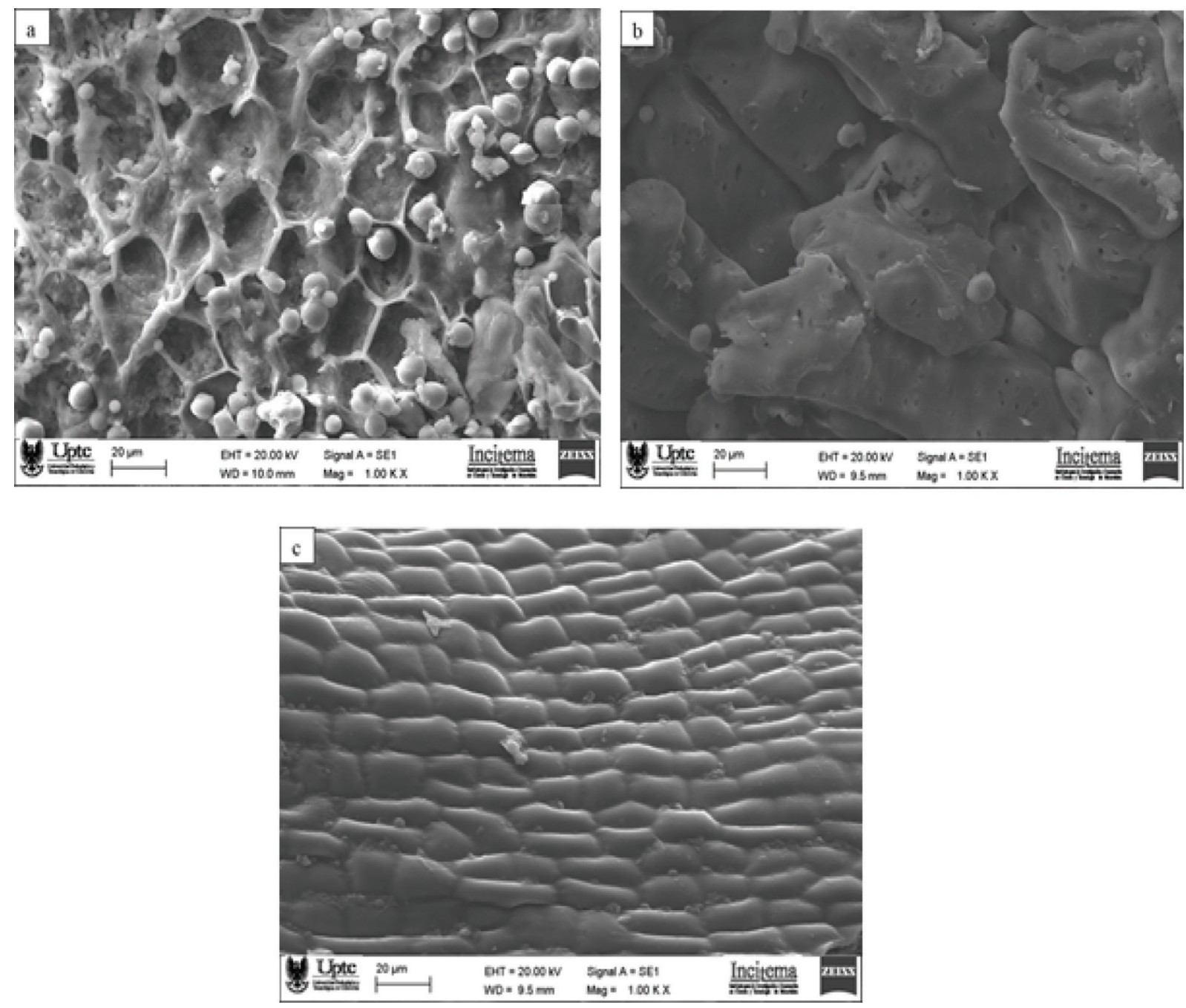

Figura 1. Escaneo de micrografías electrónicas de barrido (Escala: $20 \mu \mathrm{m}$ ): a) marlo de maíz nativo, b) marlo de maíz modificado con $\mathrm{NaOH}$ y c) marlo de maíz modificado con $\mathrm{C}_{6} \mathrm{H}_{8} \mathrm{O}_{7}$.

\section{Ensayos en reactores de lecho empacado}

La Figura (2) muestra la variación de $\mathrm{pH}$ con respecto al volumen de DAM filtrado, usando marlo de maíz nativo y modificado, donde la Figura 2(a) representa los ensayos sin lavar y la Figura 2(b) los ensayos con lavados cada $400 \mathrm{~mL}$ de efluente. En general, en los primeros $400 \mathrm{~mL}$ de DAM filtrado, se presenta un incremento de $\mathrm{pH}$ de $2.70 \pm 0.02$ a $3.29 \pm 0.10$ al utilizar marlo nativo, y a $5.14 \pm 0.10$, usando marlo modificado con $\mathrm{NaOH}$. Por el contrario, al emplear marlo modificado con $\mathrm{C}_{6} \mathrm{H}_{8} \mathrm{O}_{7}$, el pH disminuye a 2.61 \pm 0.10 . En posterior filtración (para los ensayos sin lavar) con marlo nativo y modificado con $\mathrm{NaOH}$, el $\mathrm{pH}$ disminuye y se estabiliza hacia un valor cercano al del DAM inicial, luego de los $800 \mathrm{~mL}$ alimentados, con excepción del ensayo con $\mathrm{C}_{6} \mathrm{H}_{8} \mathrm{O}_{7}$, el cual tendió a 3.0. En el caso de los ensayos con lavados, el pH del DAM disminuye drásticamente durante los siguientes $1,600 \mathrm{~mL}$. Estos resultados se comprobaron estadísticamente mediante un ANOVA que solamente existen efectos significativos en el pH del DAM filtrado a causa del tipo de marlo (con o sin modificación química) utilizado para el filtrado ( $p=3.63$ E-09). De igual forma, se verificó que no hay interacción entre estos factores y que evidentemente se presenta un mayor valor de $\mathrm{pH}$ usando marlo modificado con $\mathrm{NaOH}($ Media $=3.67)$. 

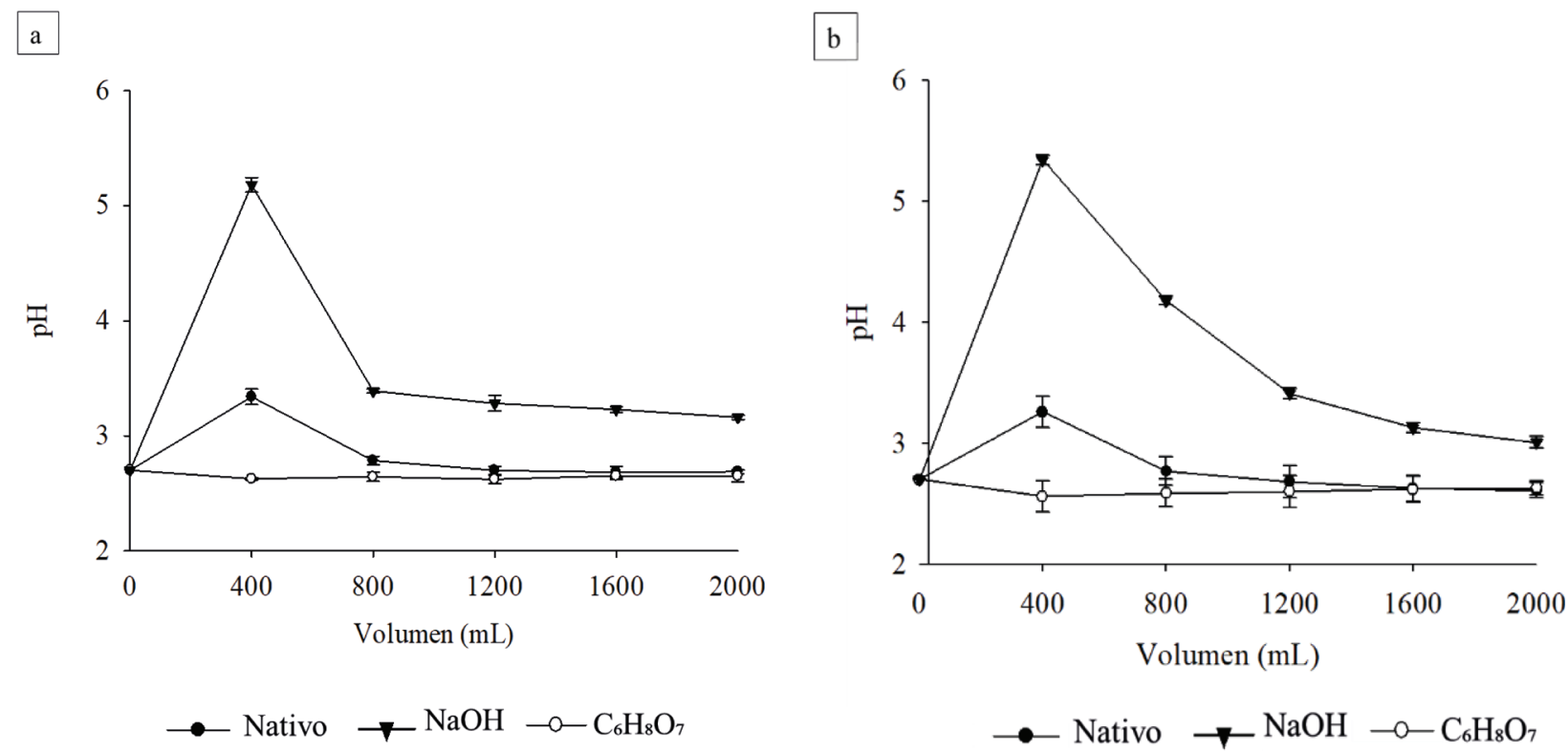

Figura 2. pH vs. volumen de DAM tratado con marlo de maíz nativo y modificado con $\mathrm{NaOH}$ y $\mathrm{C}_{6} \mathrm{H}_{8} \mathrm{O}_{7}$. (a) corresponde a los ensayos sin lavar y

(b) pertenece a los ensayos con lavados cada $400 \mathrm{~mL}$ de efluente.

Por otra parte, la Figura (3) muestra en los ensayos evaluados, la remoción de hierro (II) del DAM. En general, se observó una remoción máxima en los primeros $400 \mathrm{~mL}$ de efluente tratado, donde los ensayos con marlo modificado con $\mathrm{NaOH}$ alcanzaron los máximos valores (mayores a 50\%, con una concentración inicial de hierro en el DAM mayor a 1,000 ppm), seguidos por los ensayos con marlo nativo (alrededor del $40 \%$ con una concentración inicial de hierro en el DAM mayor a $1,000 \mathrm{ppm}$ ) y marlo modificado con $\mathrm{C}_{6} \mathrm{H}_{8} \mathrm{O}_{7}$ (entre $20 \%$ y $38 \%$ ). Sin embargo, al aumentar el volumen de DAM filtrado, la remoción de hierro disminuye en todos los casos, siendo menos notoria en los ensayos con marlo modificado con $\mathrm{NaOH}$, especialmente cuando el DAM tuvo una concentración inicial de hierro superior a 1,000 ppm tendiendo a estabilizar a un valor aproximado del $15 \%$ para los últimos volúmenes tratados (Figuras 3a y 3b). Como caso particular, los ensayos con marlo modificado con $\mathrm{C}_{6} \mathrm{H}_{8} \mathrm{O}_{7}$ y DAM a concentraciones iniciales de hierro de 500 ppm y 1,000 ppm tuvieron una drástica disminución del índice de remoción de hierro luego de filtrar $800 \mathrm{~mL}$. de DAM, lo cual pudo ocurrir por la redisolución del hierro contenido en la columna (valores de remoción negativos) (Figuras $3 b$ y $3 c$ ).

De igual manera, mediante un análisis ANOVA, se confirmó que existen diferencias estadísticamente significativas entre los niveles de concentración de hierro $(p=0.035)$ y el tipo de marlo utilizado $(p=0.048)$ en la remoción de hierro de DAM filtrado. Además, se estableció que no hubo interacción entre la concentración del DAM preparado y el marlo utilizado ( $p=0.618)$. Así mismo, se evidencia que se presenta mayor remoción de hierro en DAM con concentración inicial de hierro de 2,000 ppm (Media $=17.54 \%)$ y con marlo de maíz modificado con $\mathrm{NaOH}$ (Media $=20.49 \%$ ). 


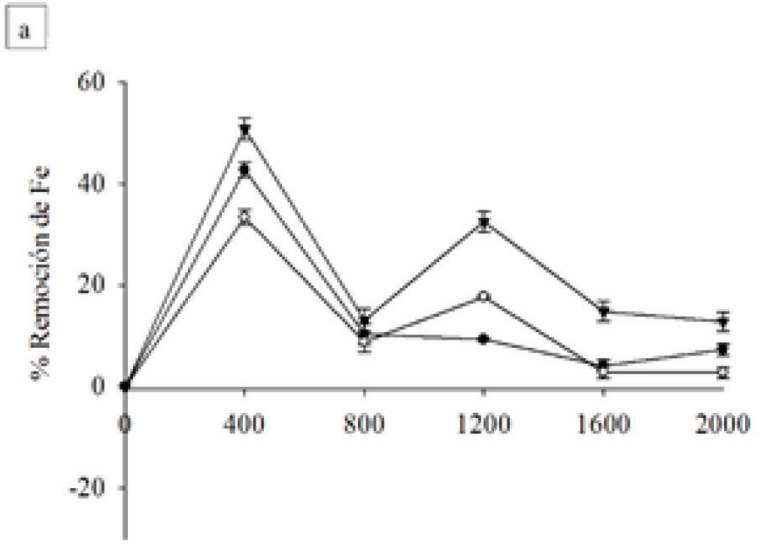

Volumen $(\mathrm{mL})$

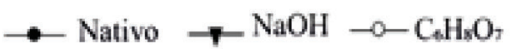

b

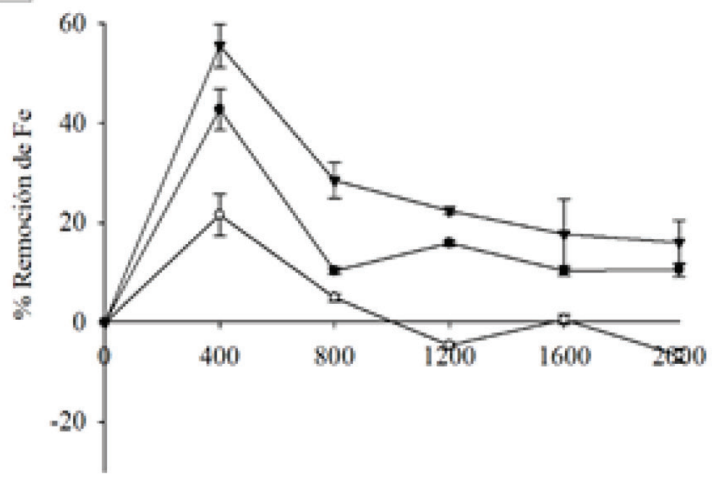

Volumen (mL)

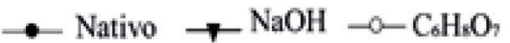

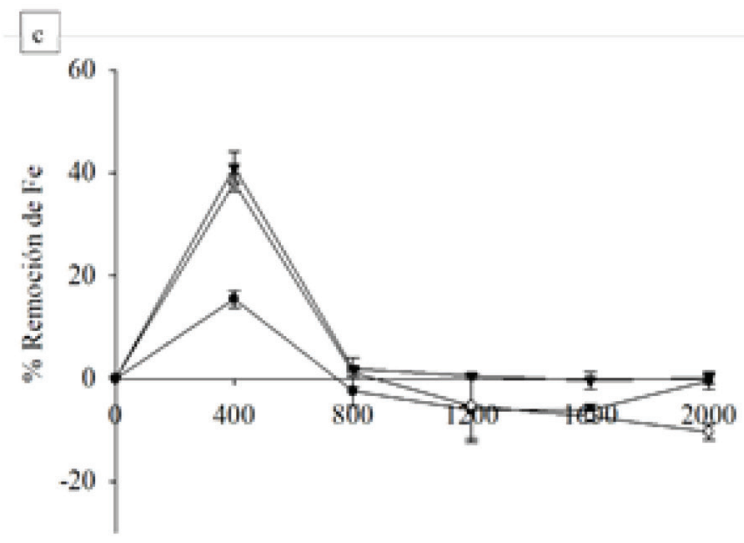

Volumen (mL)

$\rightarrow$ Nativo $\rightarrow-\mathrm{NaOH} \rightarrow \mathrm{Cs}_{3} \mathrm{O}_{7}$

Figura 3. Remoción de hierro de DAM tratado con marlo de maíz nativo y modificado químicamente con $\mathrm{NaOH}$ y $\mathrm{C}_{6} \mathrm{H}_{8} \mathrm{O}_{7}$ (ensayos sin lavar). a) 2,000 ppm, b) 1,000 ppm y c) 500 ppm.

La Figura (4) muestra los tratamientos que tuvieron los lavados del marlo de maíz cada 400 $\mathrm{mL}$ de alimentación de DAM. De la misma manera que en los ensayos sin lavados, se evidenció una máxima remoción de hierro (alrededor del $50 \%$ ) al utilizar marlo modificado con $\mathrm{NaOH}$, a concentraciones mayores de hierro de 1,000 ppm, seguido por los ensayos con marlo nativo, que lograron remociones del 40\% (Figuras 4a y $4 \mathrm{~b})$. Aunque, al aumentar el volumen de DAM la remoción disminuye, el valor se mantiene estable en los ensayos con marlo modificado, entre un $28 \pm 2 \%$ aproximadamente. Es importante resaltar como en los ensayos de marlo nativo con DAM a concentraciones de hierro de $500 \mathrm{ppm}$ se presentaron remociones del $18 \%$ y se mostró una tendencia más estable, en contraste con los ensayos sin lavado alguno (Figura 4c). Sin embargo, se demostró mediante un ANOVA que no existen efectos estadísticamente significativos en la remoción de hierro entre los niveles de concentración de hierro en el DAM ( $p=0.852)$, pero sí se presentaron diferencias significativas con el tipo de modificación del marlo $(p=0.001)$. De igual manera, se demostró que no hubo interacción entre estos factores. Es importante destacar, que el análisis confirma una mayor remoción de hierro con concentraciones de 1,000 ppm (Media $=28.06$ $\%$ ) y usando marlo modificado con $\mathrm{NaOH}$ (Media $=33.67 \%$ ). 
Angie Lorena Hernández Botia - Olga Yaneth Vásquez Ochoa - Fabio Augusto Roldan García Jairo Antonio Cubillos Lobo - Gerardo Andrés Caicedo Pineda

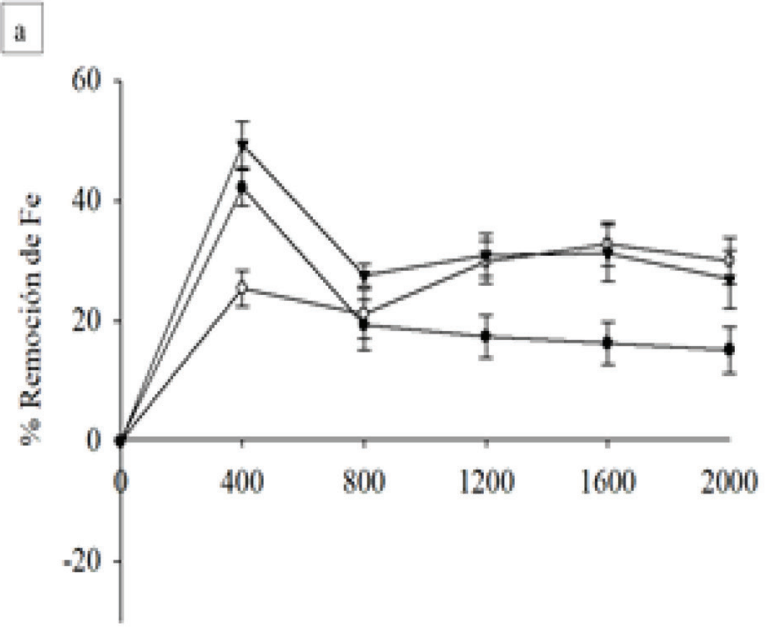

Volumen $(\mathrm{mL})$

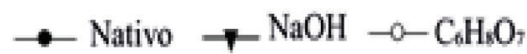

b

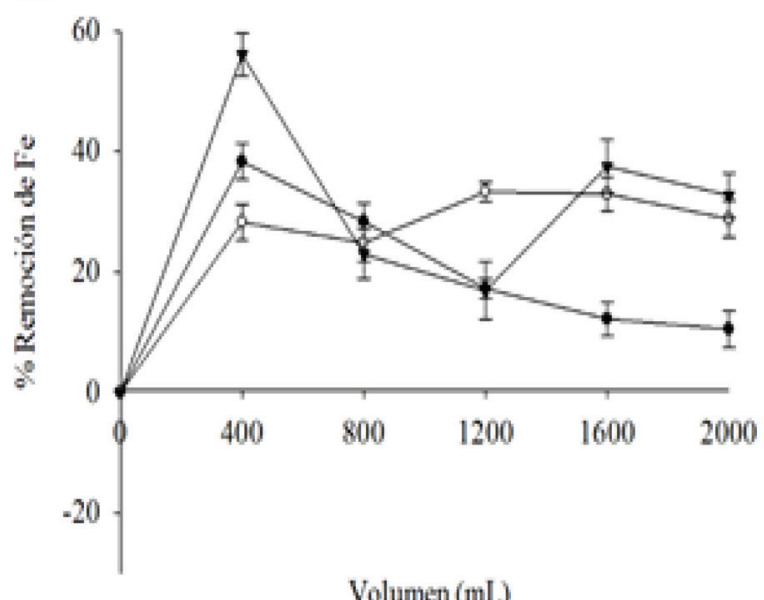

Volumen (mL)

$\rightarrow$ Nativo $\rightarrow \mathrm{NaOH} \rightarrow-\mathrm{C}_{4} \mathrm{H}_{3} \mathrm{O}_{7}$
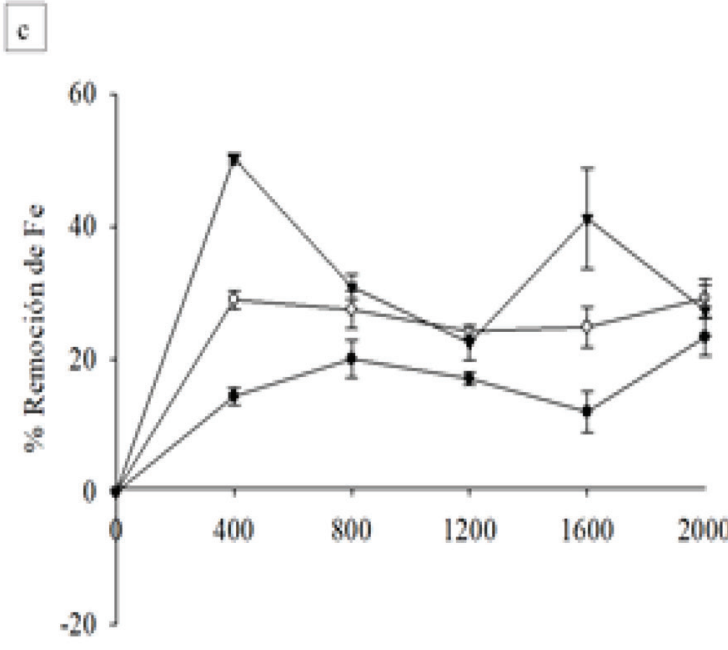

Volunen $(\mathrm{mL})$

$\rightarrow$ Nativo $\rightarrow-\mathrm{NaOH} \rightarrow \mathrm{Cs}_{s} \mathrm{O}_{7}$

Figura 4. Remoción de hierro de DAM tratado con marlo de maíz nativo y modificado químicamente con $\mathrm{NaOH}$ y $\mathrm{C}_{6} \mathrm{H}_{8} \mathrm{O}_{7}$, incluidos lavados con agua destilada cada $400 \mathrm{~mL}$ de efluente. a) 2,000 ppm, b) $1,000 \mathrm{ppm}$, c) $500 \mathrm{ppm}$.

En resumen, la remoción de hierro global con los lechos de marlo de maíz nativo y modificado se muestran en la Figura (5). Complementado a lo observado en la Figura 3, para los ensayos sin lavar, el uso de marlo modificado con $\mathrm{NaOH}$ permitió obtener las mayores eficiencias (Figura 5a), para concentraciones de hierro superiores a $1,000 \mathrm{ppm}$
(Figura 5a). En contraste, la modificación de marlo de maíz con $\mathrm{C}_{6} \mathrm{H}_{8} \mathrm{O}_{7}$ influyó negativamente sobre el proceso, disminuyendo el alcance observado para marlo nativo. Así mismo, el tratamiento de DAM con una concentración de hierro de 500 ppm, tiende a reducir su eficiencia (Figura $5 b$ ). 


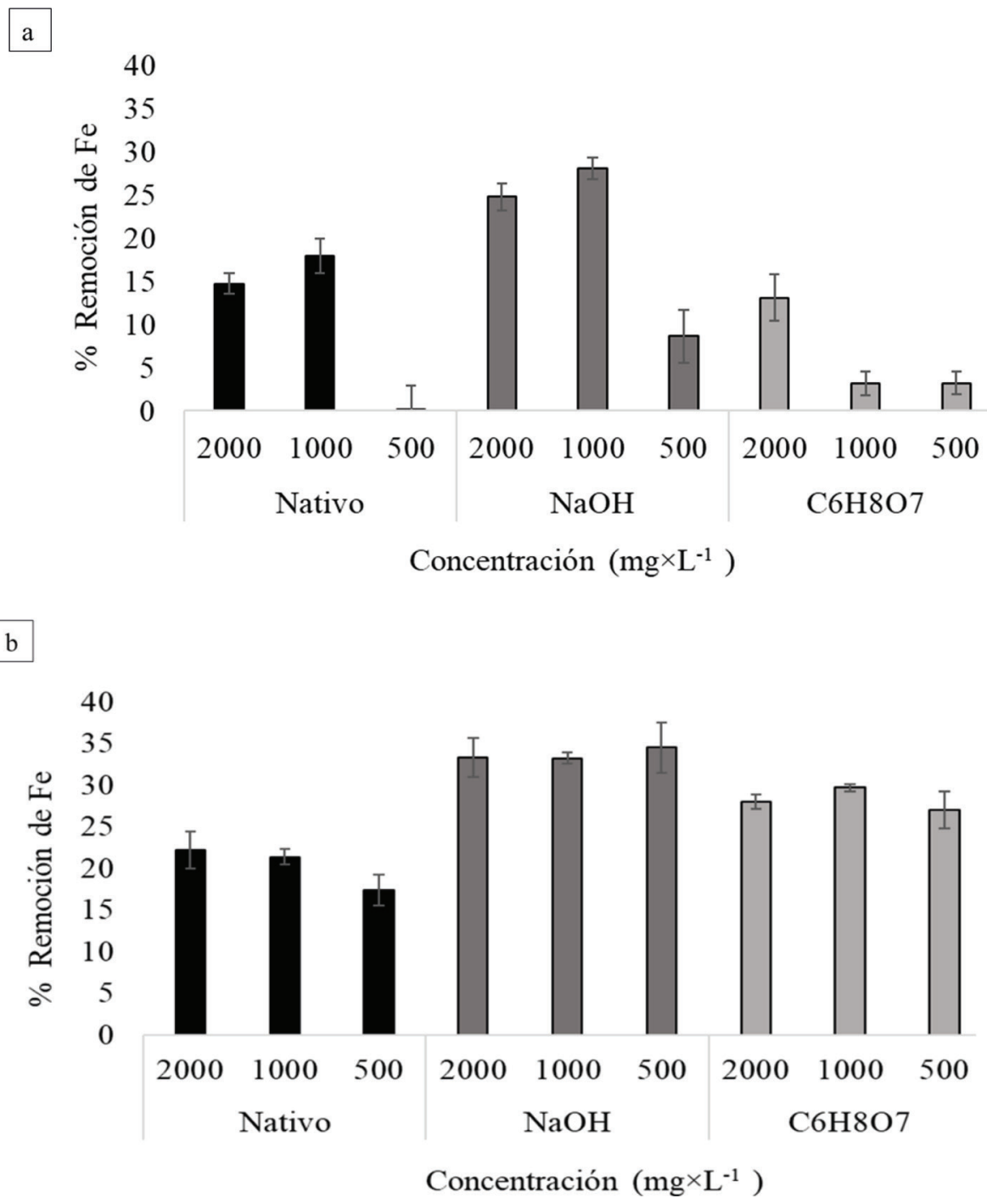

Figura 5. Remoción global de hierro, para los ensayos con marlo de maíz modificado y nativo, a diferentes concentraciones de hierro en el DAM. (a) corresponde a los ensayos sin lavar y (b) pertenece a los ensayos con lavados cada $400 \mathrm{~mL}$ de efluente.

Por otra parte, al implementar los lavados en todos los casos (Figura 5b), puede observarse un aumento notorio en la remoción y desaparición significativa de las diferencias relacionadas con la concentración de hierro, especialmente para los marlos modificados. Adicionalmente, el uso de marlo de maíz modificado con $\mathrm{C}_{6} \mathrm{H}_{8} \mathrm{O}_{7}$ permitió obtener mejores remociones que al utilizar marlo nativo, con valores aproximadamente $5 \%$ menores a los obtenidos con marlo modificado con $\mathrm{NaOH}$.

Estadísticamente se comprobó lo mencionado anteriormente, ya que se evidencian diferencias significativas en la remoción de hierro, teniendo en cuenta la concentración del metal contenido en el DAM $(p=0.039)$ y la modificación del marlo $(p=<0.001)$. De igual modo, se reitera que no hay interacción entre estos factores. A su vez, se comprueba la mayor remoción de metal en DAM con concentraciones de hierro de 2,000 ppm (Media $=22.7 \%$ ) siendo remociones muy cercanas a los valores obtenidos con DAM de 1,000 ppm (Media $=22.2 \%$ ) y se verificó que usando marlo modificado con $\mathrm{NaOH}$ (Media (Lavados) $=33.7 \% \mathrm{y}$ Media $_{(\text {Sin lavados })}=20.5 \%$ ) se obtienen los mayores porcentajes de remoción de hierro.

\section{Análisis del marlo de maíz después de la filtración de DAM}

La Figura (6) representa los espectros FTIR del marlo de maíz, donde la Figura (6a) corresponde a los espectros del marlo nativo y modificado 
con $\mathrm{NaOH}$ y $\mathrm{C}_{6} \mathrm{H}_{8} \mathrm{O}_{7}$, la Figura (6b) corresponde a los FTIR del marlo luego de los ensayos sin lavar y la Figura (6c) pertenece a los espectros FTIR luego de los ensayos con lavados cada $400 \mathrm{~mL}$ de efluente. En general, en la Figura (6a) se muestran los cambios estructurales luego de los procesos de modificación. Se observó la típica estructura lignocelulósica del marlo nativo debido a la presencia de una banda a $3,307 \mathrm{~cm}^{-1}$ atribuida a los grupos hidroxilos $(-\mathrm{OH})$ [24]. Luego de modificar el marlo con $\mathrm{NaOH}$ y $\mathrm{C}_{6} \mathrm{H}_{8} \mathrm{O}_{7}$, la señal de los grupos hidroxilo cambia a 3,333 $\mathrm{cm}^{-1}$ y $3,336 \mathrm{~cm}^{-1}$, respectivamente, lo cual se debe a la desestructuración de este grupo por los tratamientos químicos $[9,25]$. Las vibraciones de estiramiento de los grupos metilo $\left(-\mathrm{CH}_{3}\right)$ y metileno $\left(=\mathrm{CH}_{2}\right)$, son característicos de los grupos lignocelulósicos y se muestran en la banda a 2,922 $\mathrm{cm}^{-1}$, la cual no muestra efectos significativos después de la modificación química [26].

Por otro lado, se observó el pico de vibración a $1,723 \mathrm{~cm}^{-1}$ atribuido a la tensión y flexión en el plano del grupo ésteres (RCOOR'), debido al estiramiento de la vibración del carbono y el oxígeno por su alto contenido de hemicelulosa. Esta señal tiende a disminuir, al modificar con $\mathrm{NaOH}$ y se incrementa levemente modificando con $\mathrm{C}_{6} \mathrm{H}_{8} \mathrm{O}_{7}[27]$. La banda a $1,622 \mathrm{~cm}^{-1}$ es característica de la presencia de lignina y corresponde al estiramiento asimétrico del doble enlace del grupo carboxilo $(-\mathrm{COOH})$, la cual evidencia un incremento en la señal luego de las modificaciones con $\mathrm{NaOH}$ y $\mathrm{C}_{6} \mathrm{H}_{8} \mathrm{O}_{7}$ a $1,640 \mathrm{~cm}^{-1}$ y $1,636 \mathrm{~cm}^{-1}$ cuyo comportamiento se asocia principalmente a las vibraciones de flexión del agua absorbida por la celulosa [28].

Las señales a $1,510 \mathrm{~cm}^{-1}$ indican la existencia de grupos aminos $\left(-\mathrm{NH}_{2}\right)$ y se muestra un incremento considerable al modificar la biomasa con $\mathrm{NaOH}$. Las bandas a $1,420 \mathrm{~cm}^{-1}$ corresponden al estiramiento vibracional del grupo carboxílico $(-\mathrm{COOH})$, las cuales sufren un incremento en la señal al modificar con $\mathrm{NaOH}$ y se le atribuye a la posible descomposición de la lignina [29]. La banda a $1,241 \mathrm{~cm}^{-1}$ corresponde a la vibración de tensión y flexión en el plano del grupo de los ésteres, cuya intensidad de señal disminuye en ambos casos de modificación e indica que los grupos hidroxilo $(-\mathrm{OH})$ y carboxilo (-COOH) están involucrados en la incorporación del modificante en el marlo [29,30]. Finalmente, las bandas que se observan a aproximadamente $1,035 \mathrm{~cm}^{-1}$ se atribuye a la presencia de anhídridos en el marlo, esta señal disminuye con $\mathrm{C}_{6} \mathrm{H}_{8} \mathrm{O}_{7}$ como consecuencia de la formación de citratos de almidón durante el proceso de modificación [28].

Por otro lado, las Figuras (6b) y (6c), muestran que la biomasa tiende a retener su estructura composicional inicial al hacer lavados intermitentes cada $400 \mathrm{~mL}$, en contraste con los ensayos sin lavado. En el caso de los ensayos sin lavar (Figura $6 b)$, no se observaron cambios en las señales de los grupos hidroxilo $\left(3,307 \mathrm{~cm}^{-1}\right)$ y metilo $(2,922$ $\mathrm{cm}^{-1}$ ), cuando se empleó marlo nativo, indicando que hay presencia de alcoholes y metilos en la estructura al final del proceso [26]. Caso contrario sucede en las señales a $2,922 \mathrm{~cm}^{-1}, 2,848 \mathrm{~cm}^{-1} \mathrm{y}$ $1,723 \mathrm{~cm}^{-1}$ las cuales aumentan su intensidad, a causa de la formación de complejos con grupos C-H y como consecuencia de la formación de grupos anhídridos, debido a las vibraciones de estiramiento de los grupos carboxilo o carbonilo conjugados [26,28,31]. A su vez, las intensidades de las señales a $1,622 \mathrm{~cm}^{-1}$ y $1,035 \mathrm{~cm}^{-1}$ aumentan, quizás a causa de la saturación por la formación de complejos con los grupos carboxilos y anhídridos presentes en la estructura [28]. Así mismo, al usar marlo modificado con $\mathrm{C}_{6} \mathrm{H}_{8} \mathrm{O}_{7}$ no se presentaron cambios significativos en las bandas de los grupos hidroxilo, metilo y metileno, pero sí en las señales de los grupos amino $\left(1,513 \mathrm{~cm}^{-1}\right)$ y ácidos carboxílicos $\left(1,420 \mathrm{~cm}^{-1}\right)$, tal vez como consecuencia de la formación de complejos de hierro $[32,33]$. Respecto a los ensayos utilizando marlo modificado con $\mathrm{NaOH}$, en las Figuras (6b) y (6c) se evidencia una disminución en la intensidad de las señales a $3,307 \mathrm{~cm}^{-1}, 1,622 \mathrm{~cm}^{-1}, 1,513 \mathrm{~cm}^{-1}$, $1,420 \mathrm{~cm}^{-1}$ y $1,035 \mathrm{~cm}^{-1}$. En contraste con el marlo antes del proceso (Figura 6a); pueden surgir cambios posiblemente por los grupos funcionales contenidos en la lignina y celulosa, los cuales son los encargados de atrapar en la biomasa, a los cationes de hierro en solución $[4,28]$. 


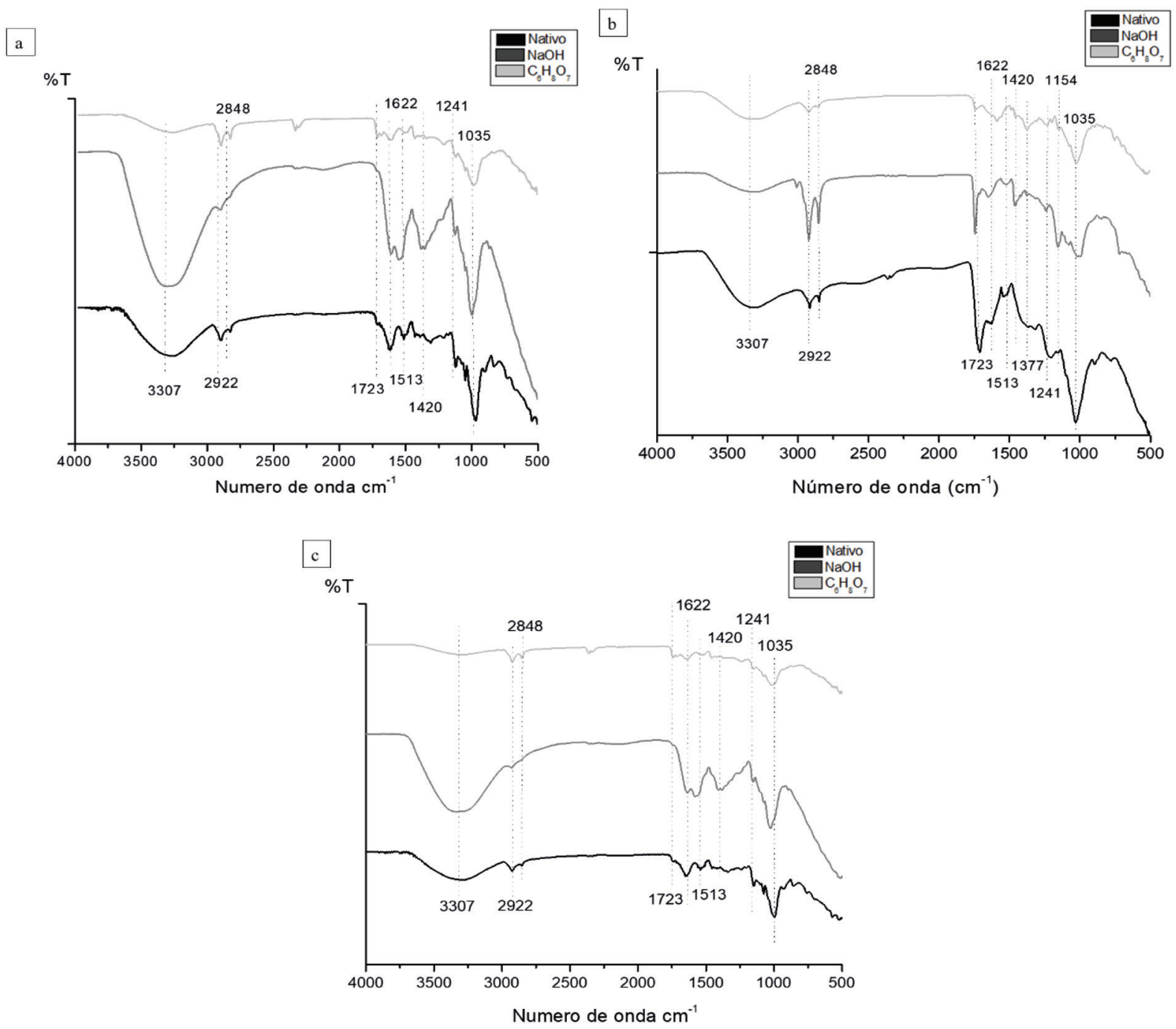

Figura 6. a) FTIR del marlo de maíz nativo y modificado, (b) FTIR luego de los ensayos sin lavar y (c) FTIR luego de los ensayos con lavados cada $400 \mathrm{~mL}$ de efluente.

\section{DISCUSIÓN}

El presente trabajo apunta a evaluar la capacidad de remoción de hierro de un marlo de maíz modificado con $\mathrm{NaOH}$ y $\mathrm{C}_{6} \mathrm{H}_{8} \mathrm{O}_{7}$ a partir de un DAM preparado a nivel de laboratorio. La modificación química del marlo de maíz se hizo con la única finalidad de conferirle propiedades morfológicas (tamaño y forma de partículas) para aumentar el área de contacto y así garantizar una destacada capacidad de remoción de hierro [33,34]. Las imágenes de la Figura (1) muestran espacios existentes entre las partículas del marlo de maíz nativo y modificado, lo cual se favorece el atrapamiento e inmovilización del metal. Por otro lado, las micrografías de las modificaciones del marlo con $\mathrm{NaOH}$ y $\mathrm{C}_{6} \mathrm{H}_{8} \mathrm{O}_{7}$ muestran estructuras heterogéneas, con imperfecciones y partículas de diferentes dimensiones, formas, tamaños regulares y fijos, resultando ser superficies apropiadas para la adsorción $[2,9,21,35]$. Wiwin et al., (2014) presentaron estructuras de marlo muy similares a este estudio. Ellos evidenciaron una estructura conformada por fibrillas con distribución desordenada y desigual, que luego de ser modificada con $\mathrm{NaOH}$ se hidroliza, resultando ser más porosa [35-38]. De igual modo, Gökben et al., (2015) muestran las micrografías resultantes de la modificación de biomasa vegetal con $\mathrm{C}_{6} \mathrm{H}_{8} \mathrm{O}_{7}$, siendo muy parecida a las obtenidas con el marlo de maíz utilizado en este trabajo, además, indican que la modificación 
ocurre principalmente en su superficie haciendo que se vuelva más esponjosa y áspera, aumentando posiblemente la capacidad de remoción de metales, puesto que es una morfología óptima para el atrapamiento de partículas [39][40].

La humedad y la densidad en el marlo aumentan con la modificación química (Tabla 1), lo cual se relacionan con el hinchamiento que le ocurre a la biomasa durante los procesos de modificación y de incorporación del $\mathrm{NaOH}$ y $\mathrm{C}_{6} \mathrm{H}_{8} \mathrm{O}_{7}[9,12]$. Morales et al., (2008) y Vargas et al., (2013) comprueban que un alto porcentaje de humedad y una baja densidad en un sistema de lecho empacado podría repercutir negativamente en el sistema, ya que puede formar aglomerados provocando el taponamiento del sistema, disminuyendo la caída de presión y el flujo libre de la disolución. Sin embargo, en esta investigación no se presentó este tipo de inconvenientes con ningún tipo de lecho, lo que se le puede atribuir al tamaño de partícula (-2 +30 \#Ty) [37- 42]. Quoc et al., (2019) por su parte indicó que la porosidad en la biomasa dentro de un sistema de lecho empacado es un parámetro muy importante, puesto que aumenta la capacidad de atrapamiento de los iones metálicos dentro de los sitios activos de adsorción. Lo anterior indica que la porosidad del marlo de maíz influye de manera positiva en la remoción del hierro, tal vez, esta propiedad influyó en gran medida en la capacidad de remoción de hierro con marlo [43].

No obstante, cabe agregar que la remoción de hierro en el DAM con marlo de maíz se ve afectada tanto por las características fisicoquímicas de la biomasa ( $\mathrm{pH}$, humedad, densidad, porosidad, tamaño, morfología y composición), como por las propiedades del metal a remover [44].

Las propiedades superficiales y el estado iónico de los grupos funcionales del marlo dependen del $\mathrm{pH}$, considerándolo como un factor control en la remoción de metales [28]. Las Figuras (2) y (6) demuestran lo expuesto anteriormente, pues, los resultados evidencian un incremento del pH en el DAM, al usar lechos con marlo nativo y modificado con $\mathrm{NaOH}$, siendo mucho más neutralizante este último, debido a la sustitución nucleofílica de grupos hidroxilos en la biomasa por la modificación (Figura 6a), la cual provoca un aumento en el pH del marlo, confiriéndole así mayor capacidad de neutralización de efluentes ácidos (Tabla 1), especialmente en los primeros 400 $\mathrm{mL}$ de DAM filtrado [11]. Sin embargo, a medida que aumenta el volumen de DAM a tratar su $\mathrm{pH}$ disminuye (entre 1 a 1.5 unidades de $\mathrm{pH}$ respecto al $\mathrm{pH}$ inicial) así como la capacidad de remoción de hierro, esto se debe a que los grupos funcionales de la biomasa tienden a neutralizarse o se cargan positivamente cuando interaccionan con el hierro (se protonan). Por esto, a medida que se aumenta el volumen de DAM a tratar su $\mathrm{pH}$ decrece hasta estabilizarse, dado que la superficie se protona y tiende a disminuir así mismo la afinidad con los cationes del metal [45].

Por otra parte, en los ensayos de marlo modificado con $\mathrm{C}_{6} \mathrm{H}_{8} \mathrm{O}_{7}$ se observó que el $\mathrm{pH}$ del DAM en los primeros $400 \mathrm{~mL}$ filtrados disminuye y luego tiende a estabilizarse al $\mathrm{pH}$ del DAM original, lo cual pudo suceder por la modificación con el $\mathrm{C}_{6} \mathrm{H}_{8} \mathrm{O}_{7}$, ya que se aumenta el contenido de $\mathrm{H}^{+}$ que se unen a los grupos funcionales, generando mayor número de sitios ácidos, además de lograr la neutralización de los grupos aminos, acidificando la biomasa y afectando la capacidad de unión entre el marlo - hierro, haciendo que se sature más rápidamente la superficie de la biomasa por el DAM filtrado $[12,42,43]$. Es importante destacar que los resultados de $\mathrm{pH}$ en los DAM filtrados no se vieron significativamente afectados por la concentración inicial del hierro en el DAM y presentaron similitud durante los ensayos con el mismo tipo de lecho, debido a que los drenajes fueron preparados con el mismo valor de $\mathrm{pH}$ (2.7).

La capacidad de remoción de hierro del marlo de maíz fue gracias a su estructura lignocelulósica y a su contenido de proteínas y carbohidratos que se componen de grupos funcionales de ácidos débiles, grupos hidroxilo y carboxílicos (Figura 6a) $[28,36]$. La remoción de hierro con marlo de maíz puede pasar por interacciones de intercambio iónico (concentrándose el hierro sobre la superficie), por fuerzas de Van der Waals o mediante la formación de enlaces en los centros activos de la biomasa $[44,45]$.

Es importante mencionar que una modificación química en la biomasa puede generar productos con alto intercambio iónico, por esto, la investigación incluyó modificaciones del marlo con $\mathrm{NaOH}$ y $\mathrm{C}_{6} \mathrm{H}_{8} \mathrm{O}_{7}[14,46]$. Luego de modificar el marlo se evidenció que se mantiene su estructura lignocelulósica debido a su amplio contenido de grupos hidroxilo, que le permitieron formar enlaces libres e intermoleculares (Figura 6a). En el caso del marlo modificado con $\mathrm{NaOH}$ se 
obtiene una estructura con alta presencia de grupos hidroxilo debido a la hidrólisis que sufrió la biomasa durante la formación de ligandos de carboxilato $\left(\mathrm{R}-\mathrm{COO}^{-}\right)$y metoxilos $\left(\mathrm{CH}_{3} \mathrm{O}^{-}\right)$, en cambio, en el marlo modificado con $\mathrm{C}_{6} \mathrm{H}_{8} \mathrm{O}_{7}$ la presencia de los grupos hidroxilos es menor debido a la formación de anhídridos cítricos y citratos de almidón [12,34]. Por su parte, Tejada et al., (2014) en su estudio acerca de la remoción de metales como $\mathrm{Pb}$, Ni y $\mathrm{Cr}$ con desechos agrícolas coincide en asignarle la capacidad de secuestrar iones metálicos por donación de un par de electrones para la formación de complejos a los grupos carboxílicos e hidroxilo [47,48].

Como caso particular, el marlo modificado con $\mathrm{NaOH}$ muestra mayor capacidad de remoción de hierro (Figura 6) debido a la reacción de deslignificación del marlo durante la modificación. El rompimiento de los enlaces de éster de la lignina y su disolución junto a la hemicelulosa genera cambios internos en la estructura del marlo, haciendo que su capacidad de hidrólisis aumente, debido a la presencia de un mayor contenido de ácidos carboxílicos y alcoholes en la pared celular del marlo de maíz [1][49]. En la Figura (6a) se evidencia la modificación del marlo con $\mathrm{NaOH}$ y se observa una mayor exposición de grupos hidroxilo y carboxilo, los cuales están involucrados en la reacción de incorporación del modificante en la biomasa, producto de la deslignificación, dicho proceso conlleva a la formación de agujeros en la superficie de la biomasa, incrementando el área superficial interna de la fracción lignocelulósica $[9,47,48]$.

Por otra parte, la remoción de hierro en DAM, utilizando marlo de maíz modificado con $\mathrm{C}_{6} \mathrm{H}_{8} \mathrm{O}_{7}$, ocurre gracias a los aniones carboxilato del grupo ácido contenido en las moléculas de aminoácidos presentes en la estructura del marlo modificado (Figura 6a), confiriéndole a la biomasa el suficiente potencial para unir iones de hierro, ya que dichos aniones se convierten en posibles sitios activos para su retención. Otras investigaciones han descubierto que la capacidad de la biomasa modificada con $\mathrm{C}_{6} \mathrm{H}_{8} \mathrm{O}_{7}$ se torna más eficiente cuando se tratan efluentes con $\mathrm{pH}$ superiores a $5[17,50,51]$. No obstante, los tratamientos llevados a cabo en los ensayos sin lavar (Figura 3) indican que la disminución en la capacidad de remoción de hierro, pudo deberse a la acidificación de la biomasa, la cual hace que se rechacen los iones metálicos de soluciones con $\mathrm{pH}$ ácido, desfavoreciendo su adsorción [45].

Reportes preliminares en literatura muestran que el $\mathrm{pH}$ del efluente influye en la capacidad de remoción de metales, ya que aumenta la posibilidad de interferir en la solubilidad de los iones, debido a la activación de los grupos funcionales de la biomasa. Así mismo, la presencia de iones $\mathrm{H}_{3} \mathrm{O}^{+}$compiten con los iones del metal por los sitios activos del marlo [2,47]. Por tal motivo, investigaciones como la de Raj et. al. (2013) buscan estrategias para aumentar el $\mathrm{pH}$ de la biomasa y desprotonizar la superficie $[8,10,11]$.

Es preciso destacar que estudios de remoción de metales con marlo de maíz nativo han demostrado capacidades adsorvativas entre el 50\% y $98 \%$ de iones metalicos como $\mathrm{Fe}, \mathrm{Pb}, \mathrm{Zn}$ y $\mathrm{Cu}$. Sin embargo, esta investigación alcanzó capacidades de remoción de hierro entre el 15\% y 30\% (Figura 3 ), incluso con marlo modificado. Esto se le puede atribuir al tipo de sistema implementado para el tratamiento, ya que, en la mayoría de las investigaciones de tratamientos de efluentes con marlo, se utilizan sistemas o reactores tipo batch o sistemas continuos con agitación, los cuales maximizan la conversión y la selectividad de las reacciones, porque se pueden controlar y predicen la conducta y las condiciones de operación (tiempo de retención hidráulica, agitación (rpm), temperatura, etc) del sistema $[16,17,28]$. En contraste, la configuración en lecho empacado permite procesar cantidades significativas de efluente, los tiempos de tratamiento son cortos y para su implementación no se necesita de altas inversiones de dinero puesto que no requiere de exigencias como grandes extensiones de tierra, parámetros de diseño, operación y mantenimiento complejos [20,52][53].

Las diferencias significativas de remoción en los ensayos sin lavar (Figura 3), respecto a la concentración de hierro en el DAM, pudo deberse a una falta de resistencia en la transferencia de masa. Probablemente, una mayor concentración permite un aumento en el número de colisiones entre el metal y la biomasa, permitiendo el proceso de adsorción de hierro [42,54]. En contraste, la más baja concentración de Fe del DAM evaluado (500 ppm) sumado a una acidificación como la que se presentó en los ensayos con marlo modificado con $\mathrm{C}_{6} \mathrm{H}_{8} \mathrm{O}_{7}$, posiblemente causaron disminución de las interacciones electrostáticas, debido a la 
ocupación metálica de los sitios activos, puesto que la biomasa modificada pudo generar agregados ácidos que limitaron la movilidad de los iones por la reducción del área superficial, generando incluso resolubilización del metal en la medida que se continuaba filtrando el efluente (Figura 6b) $[11,39,51]$. Investigaciones de otros autores corroboran lo expuesto, al mostrar que este tipo de lechos tiene potencial uso para la captura de cationes metálicos, cuando se trabaja con $\mathrm{pH}$ entre 4 y 7 , ya que a medida que el valor de $\mathrm{pH}$ crece hasta alcanzar un $\mathrm{pH}$ neutro, la superficie de la biomasa se desprotona y aumentan su afinidad con las especies catiónicas [45].

Los resultados obtenidos en los ensayos con lavados intermitentes, cada $400 \mathrm{~mL}$ de DAM filtrado (Figura 4), permitieron indicar una acción relevante del DAM sobre el marlo de maíz (nativo o modificado). Los incrementos en la remoción indican que el DAM puede afectar considerablemente los sitios activos de unión, posiblemente al ser ocupados por la alta concentración de protones en la solución ( $\mathrm{pH}$ bajos), reduciendo el área de superficie efectiva disponible para el atrapamiento de hierro $[55,56]$. Por tanto, la caída drástica de remoción, a menores concentraciones de hierro, también puede ser causada por un déficit de la fuerza direccional, encargada de superar la resistencia de transferencia de masa en el proceso de adsorción. Dicho de otra manera, si se aumenta la concentración inicial de los iones de hierro será mayor la adsorción, debido al incremento de colisiones entre el hierro y el marlo [42,57]. Además, se pudo presentar posibles disoluciones de hierro durante los filtrados, ya que el equilibrio sólido-líquido factiblemente se vería limitado por la difusión de los iones hacia los sitios activos del marlo, los cuales ya estaban ocupados o saturados [58].

Por otro lado, los ensayos que evidenciaron bajas remociones muestran que estas tienden a estabilizarse a medida que incrementa el volumen de DAM filtrado (Figuras 3 y 4). Investigadores aseguran que la remoción de metales es altamente dependiente de la concentración del metal en el efluente y que tiende a estabilizarse durante el proceso, a causa de la saturación de los sitios activos de adsorción, dificultando la interacción biomasa-metal, evidenciando así que no existe competencia significativa de los sitios activos entre el agua (solvente) y el catión $\mathrm{Fe}^{+2}[43,46]$. No obstante, los ensayos con lavados intermitentes permiten evidenciar que, de superarse los inconvenientes generados por la naturaleza de los DAM, la recuperación del lecho agotado por el proceso de remoción de contaminantes se puede llevar a cabo, simplemente con procesos de desorción física, utilizando agua, lo cual brindaría una mayor capacidad de atracción catiónica, debido al arrastre de los agregados ácidos, regenerando los sitios activos y exponiendo de mejor manera sus aniones carboxilato sobre la superficie de la biomasa (Figura 6c).

\section{CONCLUSIONES}

La interacción entre el marlo de maíz y DAM es dependiente de las propiedades fisicoquímicas de ambos componentes y de las condiciones del medio. En el caso de la biomasa, la estructura composicional lignocelulósica contiene grupos funcionales carboxílicos e hidroxilicos que favorecen la unión metal - biomasa. Los mecanismos que explican la remoción del metal son la complejación, la adsorción física y el intercambio iónico, procesos que permiten el atrapamiento de cationes en los centros activos, mediante la formación de complejos en la pared celular. Es importante agregar que, al someter el marlo de maíz a una modificación termoquímica, se logra obtener productos que pueden mejorar la capacidad de intercambio iónico, debido a la adición de grupos polares en su superficie.

En este trabajo en particular, la modificación con $\mathrm{NaOH}$ es la mejor alternativa, ya que contrarresta el efecto de la acidez de DAM. Uno de los principales factores que puede afectar negativamente la remoción de hierro, en especial cuando se trabaja con concentraciones de hierro por debajo de 1,000 ppm, debido a una competencia de los protones de la solución por los sitios activos del lecho, reduciendo el área de superficie efectiva disponible. Se pudo corroborar que dicho fenómeno sólo se podría solventar si las concentraciones de hierro superan los 2,000 ppm, debido al incremento de colisiones entre el hierro y el marlo de maíz, que mitigaría el efecto del $\mathrm{pH}$. La premisa anterior también indica el por qué la modificación con $\mathrm{C}_{6} \mathrm{H}_{8} \mathrm{O}_{7}$ no es conveniente para tratar efluentes ácidos, ya que al tener biomasa acidificada, las altas interacciones protónicas generan una repulsión que ve afectada la adsorción de los iones metálicos. 
Angie Lorena Hernández Botia - Olga Yaneth Vásquez Ochoa - Fabio Augusto Roldan García Jairo Antonio Cubillos Lobo - Gerardo Andrés Caicedo Pineda

Frente a los niveles de remoción alcanzados en los ensayos, es necesario recurrir a nueva estrategias con el fin de aumentar la capacidad de atracción metálica por los lechos, si se quisiera implementar este tipo de procesos a nivel industrial frente a DAM reales donde hay varios tipos de metales además de sulfatos. Así mismo, podría evaluarse e incluso implementarse su aplicación como pre o postratamiento de sistemas de tratamiento de DAM ya existentes, por ejemplo, en el caso de disponer altas concentraciones de metales pesados. Futuros trabajos deben considerar la evaluación de caudales de alimentación, que permitan obtener un mayor grado de remoción. También podría aplicarse en una etapa posterior a una neutralización de los efluentes.

\section{REFERENCIAS BIBLIOGRÁFICAS}

[1] C. Lavado Meza and F. Oré Jiménez, Estudio de la biosorción de plomo divalente de soluciones acuosas usando biomasa modificada de marlo de maiz (Zea mays), vol. 82, no. 4. Sociedad Química del Perú, 2016.

[2] X. Liu, Y. Liu, Z. Jiang, H. Liu, S. Yang, and Q. Yan, "Biochemical characterization of a novel xylanase from Paenibacillus barengoltzii and its application in xylooligosaccharides production from corncobs," Food Chem., vol. 264, no. November 2017, pp. 310-318, 2018.

[3] C. Duan et al., "Carbohydrates-rich corncobs supported metal-organic frameworks as versatile biosorbents for dye removal and microbial inactivation," Carbohydr. Polym., vol. 222, no. July, p. 115042, 2019.

[4] Y. Dong and J. Di, "Experimental study on the treatment of acid mine drainage by modi fi ed corncob fi xed SRB sludge particles," pp. 19016-19030, 2019.

[5] L. Luque et al., "Comparison of ethanol production from corn cobs and switchgrass following a pyrolysis - based biorefinery approach," Biotechnol. Biofuels, vol. 9, pp. 1-14, 2016.

[6] A. You, M. A. Y. Be, and I. In, "Grey water recycling with corn cob as an adsorbent Grey Water Recycling with Corn Cob as an Adsorbent," vol. 020181, no. June, 2019.
[7] A. Hormaza, "Evaluación De La Remoción De Un Colorante Azo Sobre Tuza De Maíz Mediante Diseño Estadístico," Rev. la Fac. Ciencias, vol. 1, no. 1, pp. 61-71, 2012.

[8] K. Canjura and J. Lemus, "Propuesta de un Sistema de Tratamiento para las Aguas Residuales provenientes de Lavaderos Públicos del Municipio de Nejapa," 2003.

[9] P. Piboon, N. Tippayawong, and T. Wongsiriamnuay, "Densification of Corncobs Using Algae as a Binder," C. J. Nat. Sci, vol. 16, pp. 175-182, 2017.

[10] O. Peñaranda Contreras, J. E. Perilla Perilla, and N. A. Algecira Enciso, "Revisión de la modificación química del almidón con ácidos orgánicos," Rev. Ing. e Investig., vol. 28, no. 3, pp. 47-52, 2008.

[11] N. C. Feng, X. Y. Guo, and S. Liang, "Enhanced $\mathrm{Cu}(\mathrm{II})$ adsorption by orange peel modified with sodium hydroxide," Trans. Nonferrous Met. Soc. China (English Ed., vol. 20, no. SUPPL.1, pp. s146-s152, 2010.

[12] P. Goyal and S. Srivastava, "Characterization of novel Zea mays based biomaterial designed for toxic metals biosorption," J. Hazard. Mater., vol. 172, no. June 2007, pp. 1206-1211, 2009.

[13] A. C. Morillo Coronado, Y. P. Tovar, and Y. Morillo, "Caracterización morfológica de Selenicereus megalanthus (K. Schum. ex Vaupel) Moran en la provincia de Lengupá," Ciencia En Desarrollo, vol. 7, no. 2, pp. 23-33, 2016, doi: 10.19053/01217488. v7.n2.2016.4072.

[14] K. R. Raj, A. Kardam, and S. Srivastava, "Development of polyethylenimine modi fi ed Zea mays as a high capacity biosorbent for the removal of As ( III ) and As ( V ) from aqueous system," Int. J. Miner. Process., vol. 122, no. Febrero 2013, pp. 66-70, 2013.

[15] R. E. Wing, "Corn fiber citrate: Preparation and ion-exchange properties," Ind. Crops Prod., vol. 5, no. 4, pp. 301-305, 1996.

[16] K. K. Kefeni, B. B. Mamba, and T. A. M. Msagati, "Magnetite and cobalt ferrite nanoparticles used as seeds for acid mine drainage treatment," J. Hazard. Mater., vol. 333, pp. 308-318, 2017. 
Angie Lorena Hernández Botia - Olga Yaneth Vásquez Ochoa - Fabio Augusto Roldan García Jairo Antonio Cubillos Lobo - Gerardo Andrés Caicedo Pineda

[17] Y. Nleya, G. S. Simate, and S. Ndlovu, "Sustainability assessment of the recovery and utilisation of acid from acid mine drainage," J. Clean. Prod., vol. 113, pp. 17-27, 2016.

[18] E. Peña T, A. R. Pérez R, A. J. Miranda, and J. H. Sánchez L, "Modelado de un reactor químico tipo CSTR y evaluación del control predictivo aplicando Matlab-Simulink Modeling to a CSTR reactor and evaluation of a predictive control using Matlab-Simulink," Rev. Ing. UC, vol. 15, no. 3, pp. 97-112, 2008.

[19] J. E. Franco Cogollo and D. J. Meza Cárcamo, "Evaluación de la eficiencia de un reactor de electrocoagulación a escala piloto para la remoción de algunos parámetros fisicoquímicos y microbiológicos presentes en las aguas residuales," 2017.

[20] T. Altun and E. Pehlivan, "Removal of Cr(VI) from aqueous solutions by modified walnut shells," Food Chem, vol. 132, no. 2, pp. 693 700, 2012.

[21] P. Paltán and K. Estefanía, "Comparación de la biosorción y desorción de metales pesados mediante el uso de marlo de maíz (Zea mays) en aguas contaminadas," 2018.

[22] G. J. Zagury, V. I. Kulnieks, and C. M. Neculita, "Characterization and reactivity assessment of organic substrates for sulphatereducing bacteria in acid mine drainage treatment," Chemosphere, vol. 64, no. 6, pp. 944-954, 2006.

[23] US Environmental Protection Agency, "United States Environmental Protection Agency (USEPA), 2001. Flame atomic absorption spectrophotometry. Method 7000b," no. February. Washington D.C, 2007.

[24] C. Sánchez, I. Egüés, A. García, R. LlanoPonte, and J. Labidi, "Lactic acid production by alkaline hydrothermal treatment of corn cobs," Chem. Eng. J., vol. 181-182, pp. 655660, 2012.

[25] C. Pan, S. Zhang, Y. Fan, and H. Hou, "Bioconversion of corncob to hydrogen using anaerobic mixed microflora," Int. J. Hydrogen Energy, vol. 35, no. 7, pp. 2663-2669, 2010.

[26] L. Zhang, S. Li, K. Li, and X. Zhu, "Twostep pyrolysis of corncob for value-added chemicals and high quality bio-oil: Effects of pyrolysis temperature and residence time," Energy Convers. Manag., vol. 166, no. February, pp. 260-267, 2018.

[27] D. Catherine and C. Jiménez, "Evaluación del proceso de pirólisis aplicado al material Lignocelulosico residual proveniente del pino patula en Atmosfera de dioxido de carbono," 2018.

[28] F. Oré Jiménez, C. Lavado Meza, and S. Bendezú Montes, Biosorción de Pb (II) de aguas residuales de mina usando el marlo de maiz (Zea mays), vol. 81, no. 2. Sociedad Química del Perú, 2015.

[29] N. Vilas Boas et al., "Evaluation of kinetic and thermodynamic parameters in adsorption of lead $(\mathrm{Pb} 2+)$ and chromium $(\mathrm{Cr} 3+)$ by chemically modified macadamia (Macadamia integrifolia)," Desalin. Water Treat., vol. 57, no. 38, pp. 17738-17747, 2016.

[30] C. Tejada Tovar, E. Ruiz Paternina, J. Gallo Mercado, and J. Moscote Bohorquez, "Evaluación de la biosorción con bagazo de palma africana para la eliminación de $\mathrm{Pb}$ (II) en solución," Prospectiva, vol. 13, no. 1, p. 59, Jun. 2015.

[31] N. C. León, "Caracterización de los productos obtenidos del proceso de pirolisis de residuos lignocelulósicos de agave y su uso como fuente de energía," 2017

[32] "Influence of HNO 3 oxidation on the structure and adsorptive properties of corncobbased activated carbon," vol. 41, pp. 713-722, 2003.

[33] P. Marija et al., "Removal of $\mathrm{Pb}^{2+}$ ions by raw corn silk (Zea mays L.) as a novel biosorbent," J. Taiwan Inst. Chem. Eng., vol. 0, pp. 1-10, 2015.

[34] G. A. Julisa et al., "EFECTO DEL TAMAÑO DE PARTÍCULA EN LA ADSORCIÓN DE $\mathrm{Pb}$ (II) UTILIZANDO OLOTE DE MAÍZ," no. Ii, 2015.

[35] C. C. Lugo, L. Y. V. Fiallo, and C. A. R. Reyes, "Fe and $m$ retention in natural water by adsorption-oxidation on clinoptilolite," Revista Facultad de Ingenieria, no. 66. pp. 24-44, 2013.

[36] F. Arce et al., "Chemical and structural properties of carbonaceous products obtained 
Angie Lorena Hernández Botia - Olga Yaneth Vásquez Ochoa - Fabio Augusto Roldan García Jairo Antonio Cubillos Lobo - Gerardo Andrés Caicedo Pineda

by pyrolysis and hydrothermal carbonisation of corn stover," Australian Journal of Soil Research, vol. 48, no. 7. pp. 618-626, 2010.

[37] M. Sevilla and A. B. Fuertes, "The production of carbon materials by hydrothermal carbonization of cellulose," Carbon, vol. 47, no. 9. pp. 2281-2289, 2009.

[38] K. Wiwin Rewini, I. Ishak, L. Lukman AR, and I. Hendrik, "FTIR, XRD and SEM Analysis of Microcrystalline Cellulose (MCC) Fibers from Corncorbs in Alkaline Treatment FTIR, XRD and SEM Analysis of Microcrystalline Cellulose ( MCC ) Fibers from Corncorbs in Alkaline Treatment," J. Phys., pp. 0-8, 2018.

[39] G. B. Kankiliç, A. Ü. Metin, and I. Tüzün, "Phragmites australis: An alternative biosorbent for basic dye removal," Ecol. Eng., vol. 86, pp. 85-94, 2016.

[40] M. Á. Ramírez-Niño and M. Navarro-Ramírez, "Análisis de metales pesados en suelos irrigados con agua del río Guatiquía.(Heavy Metal Analysis on Soils Irrigated with Water from the Guatiquía River)," Cienc. En Desarro., vol. 6, no. 2, pp. 167-175, 2015, doi: 10.19053/01217488.3787.

[41] M. Diana and R. Katherine, "Determinacion de la capacidad de remocion de cadmio, plomo y niquel por hongos de la podredumbre blanca inmovilizados diana milena morales fonseca katherine johanna ruiz tovar," 2008.

[42] C. L. Carolina, V. Luz Yolanda, and R. Alberto Carlos, "Remoción de Fe y Mn en aguas naturales por adsorción-oxidación sobre clinoptilolita $\mathrm{Fe}$ and $\mathrm{Mn}$ retention in natural water by adsorption-oxidation on clinoptilolite," Fac. Ing. Univ. Antioquia, vol. 66, pp. 24-44, 2013.

[43] C. Do Quoc, S. Choi, H. Kim, and S. Kang, "Application of Biosorption for Removal of Heavy Metals from Wastewater IntechOpen," Appl. Sci., vol. 9, pp. 3-15, 2017.

[44] T. Candelaria, V. Ángel, and G. Luz, "Adsorción de metales pesados en aguas residuales usando materiales de origen biológico," TecnoLogicas, vol. 18, no. 34, pp. 109-123, 2015.

[45] I. Josefina and P. Cazón, "Remoción de metales pesados empleando algas marinas," Universidad Nacional de la Plata, 2012.
[46] M. Fernández Villalón, O. Calzado Lamela, D. A. Cascaret Carmenaty, and R. M. Pérez Silva, "Factores de mayor influencia en la adsorción de metales pesados por biomasa seca de Kluyveromyces Marxianus CCEBI 2011," Tecnol. Química, vol. 38, no. 2, pp. 335-345, 2018.

[47] M. Petrović et al., "Mechanism of adsorption of $\mathrm{Cu} 2+$ and Zn2+ on the corn silk (Zea mays L.)," Ecol. Eng., vol. 99, pp. 83-90, 2017.

[48] C. Tejada, A. Villabona Ortiz, and E. Ruiz Paternina, "Study for removal of $\mathrm{Pb}$ (II), Ni (II) and $\mathrm{Cr}$ (VI) in solutions using support chemically modified," Prospect, vol. 12, no. 2, pp. 7-17, 2014.

[49] O. Sepúlveda Delgado, Z. E. Suárez Aguilar, M. Patarroyo Mesa, S. Bautista Díaz, and L. C. Canaria Camargo, "Estudio del comportamiento e impacto de la climatología sobre el cultivo de la papa y del pasto en la región central de Boyacá empleando los sistemas dinámicos in the Central Region of Boyacá Using Dynamic Systems," Cienc. en Desarro., vol. 6, no. 2, pp. 215-224, 2015.

[50] Y. Maryam Roza and V. Riku, "Tailored mesoporous biochar sorbents from pinecone biomass for the," J. Mol. Liq., vol. 291, pp. 13-20, 2019.

[51] A. González Suárez, G. Hernández Alfonso, and I. Pereda Reyes, "Pretratamiento Alcalino De Bagazo De Caña Para Mejorar La Producción De Biometano," Cent. Azúcar, vol. 46, no. 4, pp. 79-88, 2019.

[52] J. O. Nwadiogbu, V. I. E. Ajiwe, and P. A. C. Okoye, "Removal of crude oil from aqueous medium by sorption on hydrophobic corncobs : Equilibrium and kinetic studies Removal of crude oil from aqueous medium by sorption on hydrophobic corncobs : Equilibrium and kinetic studies," vol. 3655, 2018.

[53] Á. P. Sánchez Cepeda, R. Vera Graziano, E. de J. Muñoz-Prieto, E. Y. Gomez-Pachon, M. J. Bernard-Bernard, and A. M. Cerda, "Preparación y caracterización de membranas poliméricas electrohiladas de policaprolactona y quitosano para la liberación controlada de clorhidrato de tiamina," Cienc. En Desarro., vol. 7, no. 2, pp. 133-151, 2016, doi: 10.19053/01217488.v7.n2.2016.4818. 
Angie Lorena Hernández Botia - Olga Yaneth Vásquez Ochoa - Fabio Augusto Roldan García Jairo Antonio Cubillos Lobo - Gerardo Andrés Caicedo Pineda

[54] A. Albis, "Remoción de plomo de soluciones acuosas usando cáscara de yuca modificada con ácido cítrico," Av. Investig. en Ing., vol. 13, no. 1-5, 2016.

[55] Y. Vásquez, "Implementation of the passive system for coal mine drainage treatment," vol. 13, no. 128, p. 234, 2011.

[56] D. C. Ramos, "Cadmium, Copper and Lead Adsorption on Natural and Modified Bentonite, Kaolin and Zeolite: A Review of
Process Parameters, Isotherms and Kinetics in y Zeolita Naturales y Modificadas: Una Revisión de los Par ' etica," Rev. Ing., vol. 23, pp. 252-273, 2018.

[57] A. M. Lara, Caracterización y aplicación de biomasa residual a la eliminación de metales pesados. 2008.

[58] B. H. Dorian, "Bioadsorción De Metales Pesados Mediante El Uso De Biomasa Bacteriana Aislada De Jales Mineros," 2008. 